\title{
The Relationship Between Clopidogrel Gene Polymorphism to Progressive Stroke or Recurrence
}

\author{
Yuelian Lian", Xiufeng Xin ${ }^{1,2, *}$ \\ ${ }^{1}$ First Clinical Medicine School, Department of Stomatology, Jinan University, Guangzhou, China \\ ${ }^{2}$ Department of Neurology and Stroke Center, Institute of Clinical Neuroscience, Jinan University, Guangzhou, China \\ Email address: \\ 270159812@qq.com (Yuelian Lian),627141432@qq.com (Xiufeng Xin) \\ ${ }^{*}$ Corresponding author
}

To cite this article:

Yuelian Lian, Xiufeng Xin. The Relationship Between Clopidogrel Gene Polymorphism to Progressive Stroke or Recurrence. International Journal of Neurologic Physical Therapy. Vol. 6, No. 1, 2020, pp. 1-6. doi: 10.11648/j.ijnpt.20200601.11

Received: February 15, 2020; Accepted: March 2, 2020; Published: March 10, 2020

\begin{abstract}
Background: Progressive stroke is big problem what's bothering neurophysicians. CYP2C19 gene metabolism test has become more and more popular and even the routine examination of cerebral infarction patients. Objective: To evaluate the correlation of CYP2C19 gene metabolic typing, antiplatelet drug therapy to progressive stroke; to evaluate the relationship between secondary prevention compliance to recurrence of cardiovascular and cerebrovascular events. Method: Through single center and retrospectivly information collected and analyze, collect the information of gene detection of CYP2C19 in patients with acute cerebral infarction admitted within 7 days from 2012 to 2016 and used clopidogrel, antiplatelet drug therapy, past history and others to analyze the related factors to progressive stroke; through regular telephone or outpatient follow-up to evaluate the relationship between secondary prevention compliance to recurrence of cardiovascular and cerebrovascular events. Result: A total of 436 patients were collected. CYP2C19 fast metabolism type $(* 1 / * 1)$ accounted for $39.22 \%$, medium metabolism type $(* 1 / * 2$ and $* 1 / * 3) 50.92 \%$, slow metabolism type $9.86 \%(* 2 / * 3, * 2 / * 2$ and $* 3 / * 3)$. Patients with good secondary prevention compliance had fewer cardiovascular and cerebrovascular recurrence events. There's no significant correlation between the choice of loading dose of clopidogrel or combination of antiplatelet therapy to progressive stroke and cardiovascular or cerebrovascular events after 3 years of stroke. Conclusion: Limited to retrospective analysis and number of cases in a single center, further research to enlarge the number of centers and cases is needed.
\end{abstract}

Keywords: Clopidogrel, Progressive Stroke, CYP2C19

\section{Introduction}

With the rapid development of medical technology, in addition to intravenous thrombolysis, there are also stent thrombectomy and bridging therapy for recanalization of vessels in acute phase of cerebral infarction. However, for most patients, oral drugs such as antiplatelet and others which can minimize the progression or recurrence of cerebral infarction, are also very important in acute and secondary prevention stage. As the second generation of thiophene pyridine antiplatelet drugs, clopidogrel is widely used in the prevention and treatment of cardiovascular and cerebrovascular diseases. It is widely recommended in the domestic and foreign guidelines for the use of acute and secondary prevention of ischemic cerebrovascular diseases
[1-3]. The gene polymorphism involved in the drug metabolism of clopidogrel is an important factor to prevent the progressive or recurrence of stroke. Clopidogrel is an inactive drug precursor, it needs to be activated by CYP450 metabolizing enzyme in the liver to interact with P2Y12 receptor on the platelet surface [4]. CYP2C19 (cytochrome P450, family 2, subfamily C, polypetide 19) is the key enzyme for the metabolism of clopidogrel. CYP2C19 gene polymorphism is mainly caused by two kinds of mutations: one is to enhance enzyme activity, the other is to reduce enzyme activity. The response of patients to clopidogrel is different, some patients who take clopidogrel for a long time may have recurrence of ischemic stroke and other vascular events, This phenomenon is called clopidogrel resistance clinically [5]. The gene polymorphism of clopidogrel is critical to resistance [6] and gene polymorphism can provide 
basis for clinical antiplatelet drug selection [7]. In different studies, the incidence of clopidogrel resistance varied obviously, Gurbel PA and other studies suggest that the incidence of clopidogrel resistance varies from $5 \%$ to $44 \%$ [8], Fiolaki [9] found that the incidence of clopidogrel resistance was $27 \%$ in a meta-analysis.

\section{Methods}

\subsection{Design Overview}

This study was based on a single center and retrospective study. All cases were from the neurology department of the first affiliated hospital of Jinan University. Patients with acute ischemic stroke admitted to hospital within 7 days of onset from January $1^{\text {st }} 2012$ to December $31^{\text {st }} 2016$ were collected. All of the patients should fulfill genotyping of CYP2C19.

\subsection{Participants}

Over 18 years of age; The diagnosis of cerebral infarction in patients with acute cerebral infarction within 7 days of onset was in accordance with the diagnostic criteria of cerebral infarction in the national guidelines for the prevention and treatment of cerebrovascular disease in 2014 [10] and was confirmed by CT / MRI. All of the patients should use clopidogrel and fulfill CYP2C19 gene detection. Subjects were excluded if they underwent emergency thrombolytic therapy or intravascular therapy; Patients who received anticoagulant drugs within 7 days of admission included low molecular weight heparin (LMWH); Patients with asymptomatic cerebral infarction were found after admission; Patients enrolled in clinical studies; Patients with previous cerebral infarction were treated with antiplatelet drugs within 2 weeks.

\subsection{Methods of Data Collection}

The electronic medical records included "cerebral infarction", "atherosclerotic cerebral infarction", "cardiogenic cerebral embolism", "lacunar infarction" and "cerebrovascular disease" according to the diagnosis of discharge from January 1 st 2012 to December 31st 2016.

\subsubsection{Antiplatelet Drug Selection}

NIHSS score $\leq 7$ points: loading dosage of clopidogrel 300 $\mathrm{mg}$ for the $1^{\text {st }}$ time $(75 \mathrm{mg}$ qd from the next day) and aspirin 100mg QD; determined according to specific circumstances. If the patient has previous gastric hemorrhage and other intolerant double antibodiesa history of cerebral infarction, coronary heart disease, They were given aspirin $100 \mathrm{mg} / \mathrm{d}$ temporarily, clopidogrel $75 \mathrm{mg} / \mathrm{d}$ from the second day, and Clopidogrel / aspirin was given according to ESSEN score after 7 days.

NIHSS score $\geq 8$ points: according to the ESSEN score, clopidogrel (first time of $300 \mathrm{mg}$ loading dose and then $75 \mathrm{mg}$ QD) or aspirin 100mg QD was given.

\subsubsection{Clinical Follow-up}

The first follow-up was carried out 3 months after discharge through outpatient service and telephone follow-up. The medication choice, the occurrence and time of the end event were recorded in detail. Telephone or outpatient follow-up will be conducted at $6,12,24$ and 36 months after discharge. The final deadline for follow-up was December $31^{\text {st }} 2019$.

\subsubsection{The Clinical End Points of Cardiovascular and Cerebrovascular Events}

Recurrence of ischemic stroke, vascular death and myocardial infarction.

\subsubsection{Main Outcome Endpoint Events}

Early recurrence or progression: NIHSS scores after admission to 7 days were higher than baseline $\geq 2$ points. Refer to the multicenter clinical study standards of CLASS-CHINA [11].

\subsubsection{CYP2C19 Detection Method and Judgment Criteria}

CYP2C19 gene detection kit was determined by DNA microarray method. Blood samples (venous blood, no less than $2 \mathrm{ml}$ ) were collected from EDTA anticoagulant tube on the second day after admission, and then sent for examination on the same day. CYP2C19 genotype was detected from genomic DNA extracted from peripheral blood. In this kit, CYP2C19 gene specific pair primers 1 and 2 were amplified by PCR, a specific hybridization reaction was carried out between the amplification product labeled with biotin and the CYP2C19 gene type detection probe fixed on the aldehyde substrate. The specific hybridization signal is colored by enzymatic color reaction. By scanning the chip, the hybridization image of the sample DNA amplification product and the wild-type and mutant probes of each gene site is obtained. Through analyzing the image by software to judge the genotype of the sample be tested. The main components of the assay include: CYP2C19 amplification solution 1, CYP2C19 amplification solution 2, reaction solution A, reaction solution $\mathrm{B}, \mathrm{CYP} 2 \mathrm{C} 19$ gene chip, CYP2C19 positive control solution, CYP2C19 negative control solution and baio hybrid color development kit. The result is one of six genotypes: CYP2C19*1/*1, CYP2C19*2/*2, CYP2C19*3/*3, CYP2C $19 * 1 / * 2, \quad$ CYP2C19*1/*3, CYP2C19*2/*3. CYP2C19*1 represents no base mutation with normal enzyme activity; CYP2C19*2 represents $681 \mathrm{G}>\mathrm{A}$ base mutation without enzyme activity ; CYP2C19*3 represents 636 $\mathrm{G}>$ Abase mutation without enzyme activity. CYP2C19*1 is a gene encoding the activity of normal metabolic enzymes, CYP2C19*2 and CYP2C19*3 is the main mutation type in the Han population。There are three corresponding phenotypes, they are rapid metabolic type (CYP2C19*1/*1 type with two mutation sites of enhanced enzyme activity), intermediate metabolic type (CYP2C19*1/*2 and CYP2C19*1/*3 types with only one mutation site that reduces or enhances enzyme activity) and slow metabolic type (CYP2C19 *2/*3, CYP2C19 *2/*2 and CYP2C19 *3/*3 types with two mutation sites reducing enzyme activity).

\subsection{Statistical Methods}

Statistical Analysis of data by 9.3 software. Data is expressed 
as the count and percentage of classified variables, as the average and standard deviation of continuous variables (SD). T test is used to evaluate the difference of mean value of normal distribution variables. For the variables with non normal distribution, based on Wilcoxon's rank sum test, two sets of $T$ tests with different means after logarithmic transformation are used. $X 2$ test was used to test the difference of binary variables and the incidence of the main end point events (vascular death, stroke recurrence and acute myocardial infarction) during the follow-up period. and the type of gene polymorphism and the relationship between other factors and the main end point events were analyzed by logistic analysis. The statistical significance was explained as $P<0.05$.

\section{Results}

\subsection{General Information}

From the baseline data table 1, it can be seen that the older the patient is, the more serious the neurological deficit is at the time of admission (the higher NIHSS and mRS scores), the larger the area of cerebral infarction and the patients admitted within 24 hours are more likely to have progressive stroke.

Table 1. Baseline data with progressive stroke.

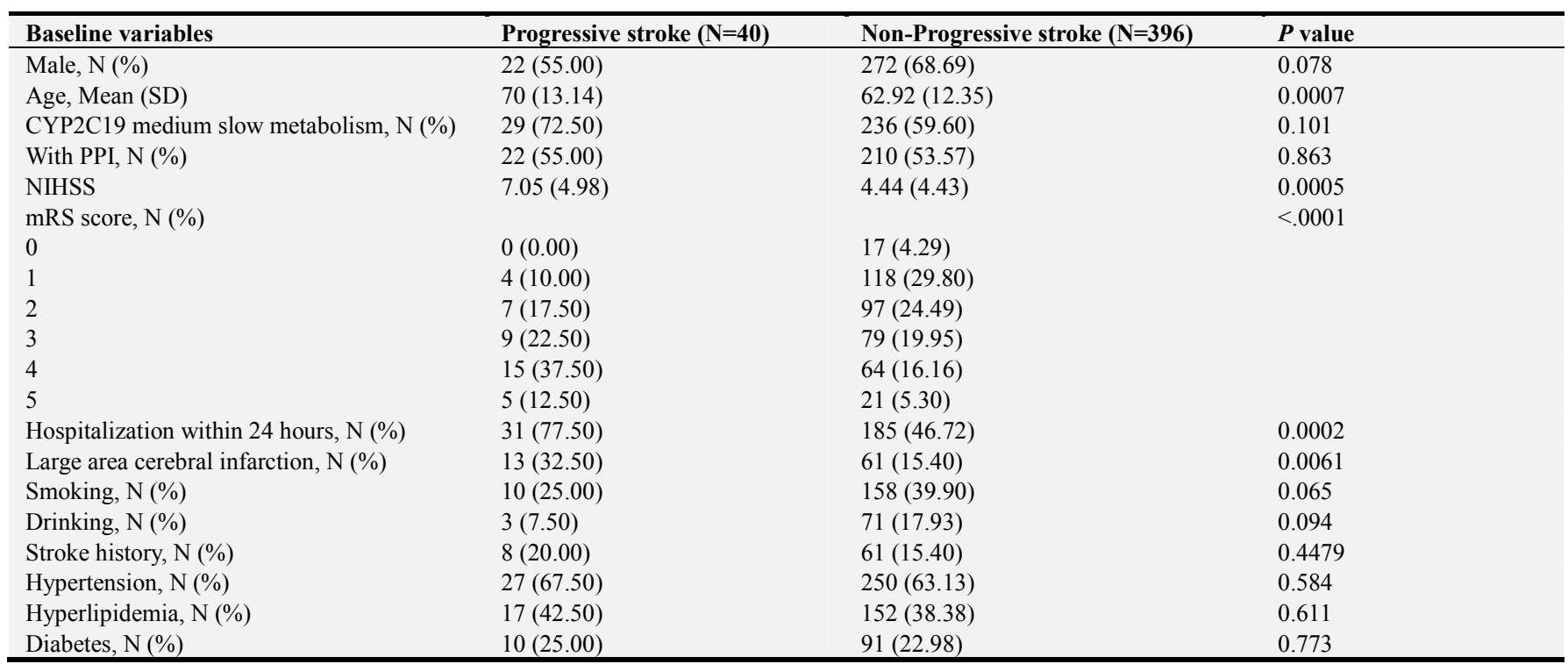

\subsection{CYP2C19 Gene Distribution}

From 2012 to 2016, 436 patients with acute cerebral infarction admitted to hospital within 7 days of onset and fulfilled CYP2C19 gene detection were selected. 171 cases $(39.22 \%)$ of CYP2C19*1/*1 type, 176 cases $(40.37 \%)$ of CYP2C $19 * 1 / * 2$, 46 cases $(10.55 \%)$ of CYP2C $19 * 1 / * 3,18$ cases $(4.13 \%)$ of $\mathrm{CYP} 2 \mathrm{C} 19 * 2 / * 2,17$ cases $(3.9 \%)$ of
CYP2C19*2/*3 and 8 cases $(1.83 \%$ ) of CYP2C19*3/*3. Figure 1 shows CYP2C19 fast metabolic type accounted for $39.22 \%$, intermediate metabolisc type $50.92 \%$, slow metabolic type $9.86 \%$. A total of 40 patients developed progressive stroke and among these progressive stroke patients intermediate and slow metabolic type of CYP2C19 genotype takes for $62.5 \%$. There shows to be certain correlation between CYP2C19 genotype metabolic type and progressive stroke.

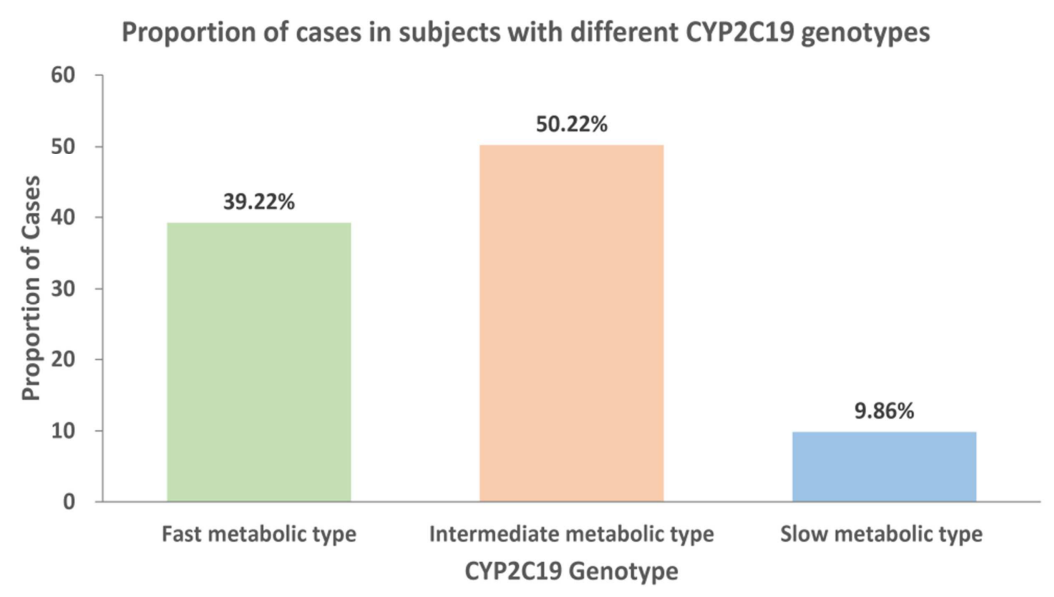

Figure 1. Proportion of cases in subjects with different CYP2C19 genotypes. 


\subsection{Antiplatelet Therapy and CYP2C19 Metabolism in Patients with Progressive Stroke}

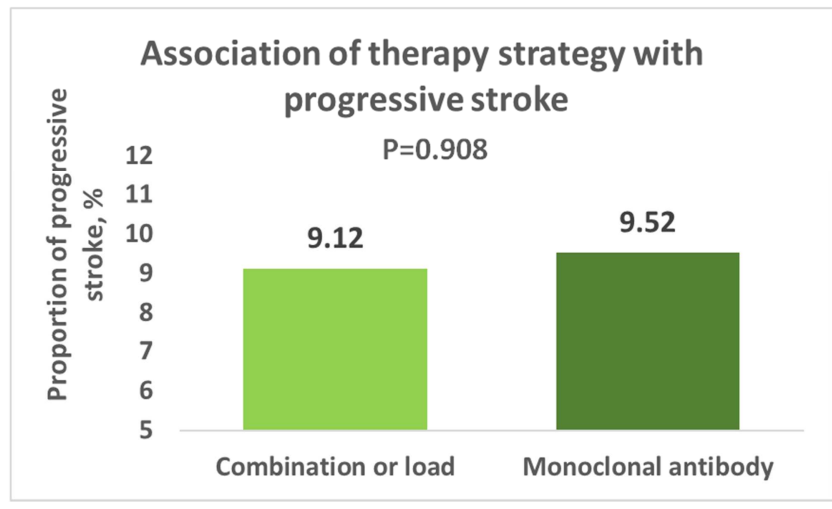

Figure 2. Antiplatelet therapy and progressive stroke.

A total of 351 patients chose loading dosage or combined antiplatelet treatment, 32 patients had progressive stroke. 85 patients were not treated with loadindg dosage or combined antiplatelet treatment, 8 patients had progressive stroke. As we can see from Figure 2, there is no statistical difference in the use of loadindg dosage or combined antiplatelet treatment.

\subsection{Analysis of Related Factors to Progressive Stroke}

As can be seen from table 2, univariate logistic regression statistical analyses shows that NIHSS and mRS scores at admission, admission within 24 hours after onset of disease and large area cerebral infarction were significantly related to the occurrence of progressive stroke. There's no significant correlation with progressive stroke among CYP2C19 genetype of intermediate or slow matabolic type and loadindg or combined antiplatele therapy. Multivariate logistic regression analyses also indicated that there was a correlation between NIHSS score at admission within 24 hours after onset to progressive stroke.

Table 2. Univariate logistic regression analyses and multiple logistic regression analyses.

\begin{tabular}{|c|c|c|c|c|}
\hline \multirow{2}{*}{ Predictors } & \multicolumn{2}{|c|}{ Univariate logistic regression analyses } & \multicolumn{2}{|c|}{ Multiple logistic regression analyses } \\
\hline & OR & $P$ & OR & $P$ \\
\hline Male & $1.8(0.93-3.47)$ & 0.0816 & & \\
\hline CYP2C19 intermediate or slow type & $1.79(0.87-3.68)$ & 0.1152 & & \\
\hline Age & $1.05(1.02-1.08)$ & 0.0009 & & \\
\hline NIHSS & $1.1(1.04-1.16)$ & 0.001 & & \\
\hline $\mathrm{mRS}$ score & $1.74(1.34-2.26)$ & $<.0001$ & $1.58(1.12-2.23)$ & 0.009 \\
\hline 24-hour hospitalization & $3.93(1.82-8.46)$ & 0.0005 & $2.78(1.03-7.51)$ & 0.044 \\
\hline Smoking & $0.5(0.24-1.06)$ & 0.0693 & & \\
\hline Drinking & $0.37(0.11-1.24)$ & 0.1068 & & \\
\hline Stroke history & $1.37(0.6-3.12)$ & 0.4489 & & \\
\hline Large infarction & $2.65(1.29-5.41)$ & 0.0077 & & \\
\hline Hypertension & $1.21(0.61-2.42)$ & 0.5848 & & \\
\hline Hyperlipidemia & $1.19(0.61-2.29)$ & 0.6109 & & \\
\hline Diabetes & $1.12(0.53-2.37)$ & 0.773 & & \\
\hline PPI & $1.06(0.55-2.04)$ & 0.863 & & \\
\hline loadindg or combined antiplatelet & $0.95(0.42-2.15)$ & 0.9077 & & \\
\hline compliance & $0.46(0.18-1.16)$ & 0.1006 & $0.42(0.16-1.09)$ & 0.0754 \\
\hline
\end{tabular}

\subsection{The Relationship Between Compliance of Secondary Prevention of Cerebrovascular Disease to Cardiovascular and Cerebrovascular Events}

All patients were followed up regularly to evaluate the relationship between the compliance of secondary prevention drugs and the occurrence of cardiovascular and cerebrovascular events. 306 patients were followed up successfully at the 3-year cut-off point, and the success rate was $70.2 \%$. There were 235 patients with good compliance of secondary prevention medication and 13 patients with progressive stroke, 71 patients with poor compliance and 8 patients with progressive stroke. From figure 3, there is no statistical difference to compliance. Through figure 4 , we can see that the survival curve analysis of compliance of secondary prevention shows that there are obvious differences between them, but still has no statistical difference.

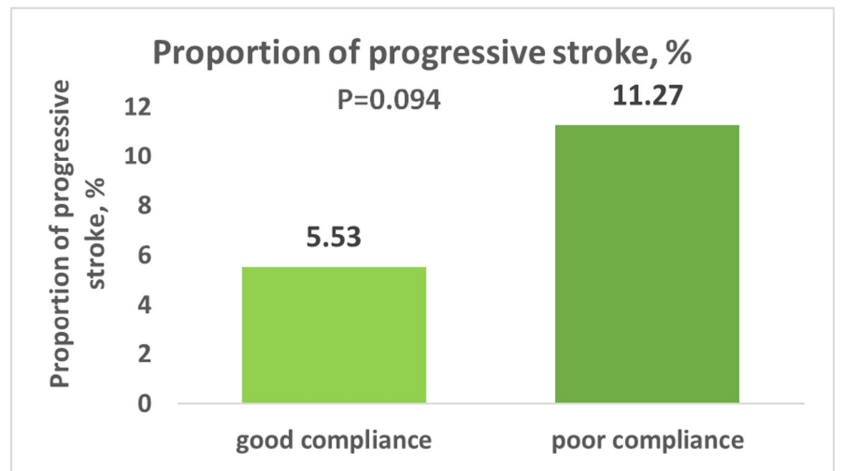

Figure 3. Secondary prevention compliance to recurrence of cardiovascular and cerebrovascular events. 


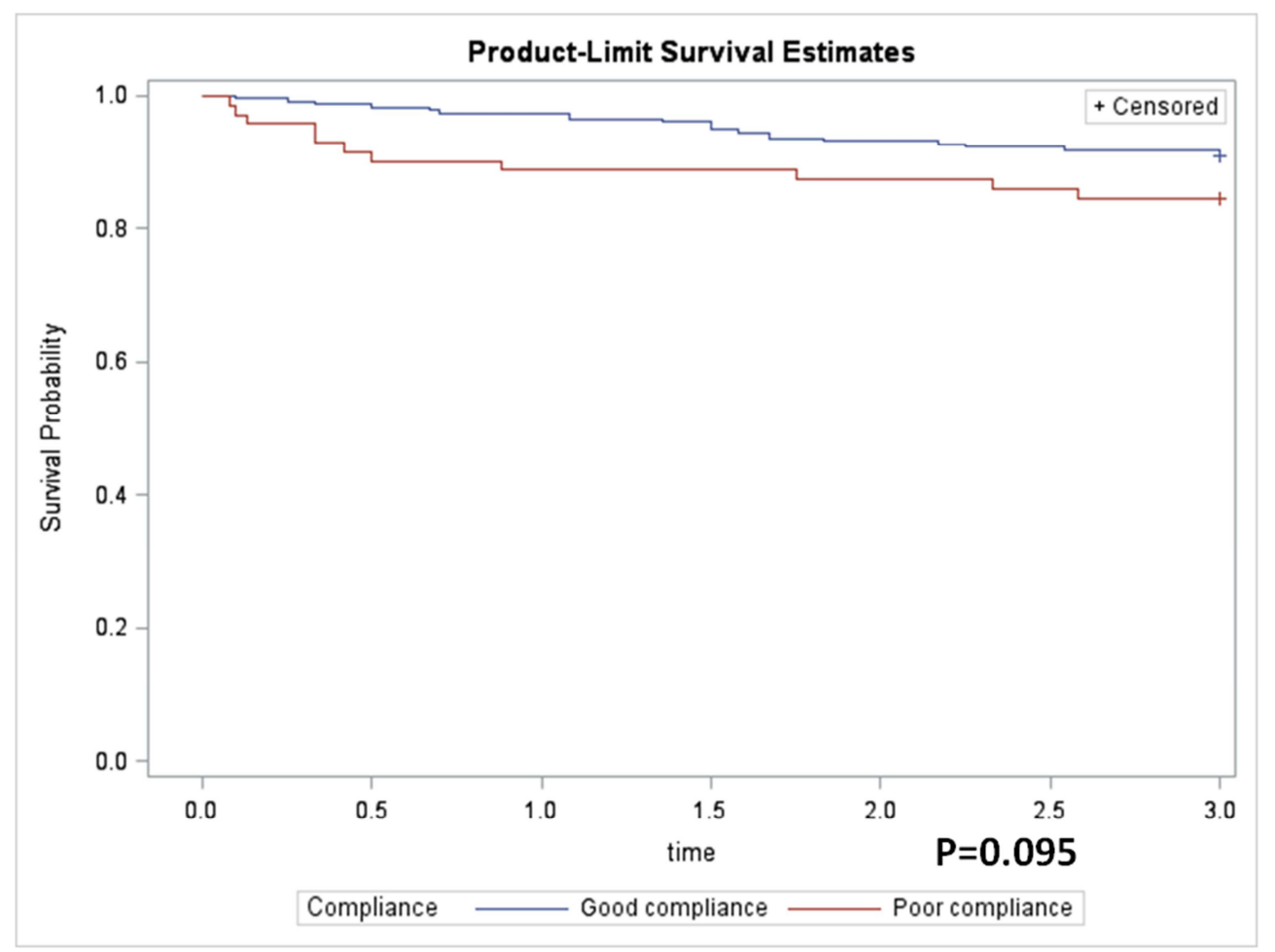

Figure 4. Analysis of secondary prevention compliance and survival evaluation.

\section{Discussion}

Clopidogrel has no biological activity. As a precursor drug, it is metabolized into active product by CYP2C19 enzyme in the liver, and plays a role in inhibiting platelet activation and aggregation. At present, they are mainly known as CYP2C19, CYP3A4, CYP3A5, CYP2C9 and CYP2B6. The key enzyme in the metabolism of clopidogrel is generally believed to be CYP2C19. CYP2C19 is mediated by CYP2C19 gene on chromosome 10, which is the most important enzyme in metabolism and can transform about $40 \%$ of clopidogrel in liver. CYP2C19 $* 1$ is a wild-type allele encoding normal metabolic enzyme activity, while CYP2C19*2 and CYP2C19 *3 have multiple single nucleotide polymorphisms, which are also the major mutant alleles in the Han population. At present, some research suggest that patients with CYP2C19 gene polymorphism in the medium and slow metabolism types (CYP2C19 *2 and CYP2C19*3) are more likely to have stroke progression and recurrence in clinic due to the influence of antiplatelet drug clopidogrel aggregation reaction, which is competent for low response or resistance of clopidogrel.

The intermediate metabolic type of CYP2C19 patients in our center takes up the largest proportion $(50.92 \%)$, the proportion besides fast metabolic type in CYP2C19 patients with progressive stroke was also the highest $(70 \%)$. The frequency of fast metabolic, intermediate metabolisc and slow metabolisc in all patients were $39.22 \%, 50.92 \%$ and $9.86 \%$ respectively, which was close to the distribution of Zhang and Han's research $[12,13]$. It can be seen that the low response or resistance of clopidogrel is related to progressive stroke. For patients with low response or resistance to clopidogrel, whether there is a more appropriate choice of antiplatelet drugs is worthy of further study and selection by basic and clinical doctors.

The activity of CYP2C19 is affected by many kinds of drugs. Different types of proton pump inhibitors, especially omeprazole, have the strongest effect on the antiplatelet effect of clopidogre. The proton pump inhibitor is the substrate of CYP2C19, which can competitively inhibit the metabolism of clopidogrel. Gilard Carried out a prospective randomized controlled trial in which patients after percutaneous coronary intervention were given omeprazole and placebo respectively under the condition of using clopidogrel combined with aspirin as antiplatelet precursors, and the platelet activity was measured. The results suggested that omeprazole could affect the clinical efficacy of clopidogrel [14]. A large cohort study published by juurlink showed that patients with acute myocardial infarction treated with clopidogrel and proton pump inhibitors had a $27 \%$ increased risk of reinfarction within 90 days [15]. The relationship between proton pump inhibitors and progressive stroke worth further analyze in our study.

According to the baseline data, there was a significant correlation between progressive stroke and the degree of neurological deficit (the higher NIHSS and mRS scores), whether it was large area of cerebral infarction and the time of admission (whether it was within 24 hours after the onset at the time of admission). It suggested that patients after onset of clinic need to be monitored for nervous function as early as possible so that clinicians can deal with clinical changes as soon as possible. Chance study [16] has confirmed the superiority of dual antiplatelet drug selection in minor stroke 
and high-risk transient ischemic attack patients. In this study, the advantages of loading dose of clopidogrel and dual antiplatelet were not obvious. It worth further expanding the sample size study.

Follow up work plays an important role in tracking the prognosis of patients, secondary prevention compliance, recurrence of cardiovascular and cerebrovascular events of myocardial infarction. The long-term secondary prevention compliance of patients with clopidogrel plays a decisive role in reducing the recurrence of cardiovascular and cerebrovascular events. In this study, limited to the sample size and follow-up period (3 years after stroke), the corresponding results were not statistically significant. It worth further research to expand the sample size or increase the follow-up period.

\section{Conclusion}

It plays an important role in the comprehensive management of patients, such as the necessary auxiliary examination of CYP2C19 genotyping, the selection of appropriate antithrombotic programs for patients, the management of acute phase and the follow-up work of long-term secondary prevention period. This study has some limitations, which need to be further confirmed by expanding the sample size, multicenter, long-term follow-up control experimental study.

\section{References}

[1] Chinese Medical Association Neurology Society and Chinese Medical Association Neurology Branch of Cerebrovascular Disease. Guidelines for secondary prevention of ischemic stroke and transient ischemic attack in China 2014 [J]. Chinese Journal of Neurology, 2015, 48 (4): 258-273.

[2] Chinese Medical Association Neurology Society and Chinese Medical Association Neurology Branch of Cerebrovascular Disease. Guidelines for the diagnosis and treatment of Acute Ischemic Stroke in China 2018 [J]. Chinese Journal of Neurology, 2018, 51 (9): 666-682.

[3] Powers WJ, Rabinstein AA, Ackerson T, et al. 2018 Guidelines for the Early Management of Patients With Acute Ischemic Stroke: A Guideline for Healthcare Professionals From the American Heart Association/American Stroke Association [J]. Stroke, 2018, 49 (3): e46.

[4] Pereira NL, Geske JB, Mayr M, et al. Pharmacogenetics of clopidogrel: an unresolved issue [J]. Circ Cardiovasc Gene, 2016, 9 (2): 185-188.

[5] Sofi F, Marcucci R, Gori AM, et al. Clopidogrel non-responsiveness and risk of cardiovascular morbidity. An updated meta-analysis [J]. Thromb Haemost, 2010, 103 (4): 841-848.

[6] Lewis JP, Shuldiner AR. Clopidogrel pharmacogenetics: Beyond candidate genes and genome- wide association studies [J]. Clin Pharmacol Ther, 2017, 101 (3): 323-325.

[7] Amin AM, Sheau Chin L, Azri Mohamed Noor D, et al. The personalization of clopidogrel antiplatelet therapy: the role of integrative pharmacogenetics and pharmacometabolomics $[\mathrm{J}]$. Cardiol Res Pract, 2017, 8062796.

[8] Gurbel PA, Tantry US. Clopidogrel resistance?[J]. Thromb Res, 2007, 120 (3): 311-321.

[9] Fiolaki A, Katsanos AH, Kyritsis AP, et al. High on treatment platelet reactivity to aspirin and clopidogrel in ischemic stroke: a systematic review and meta-analysis [J]. J Neurol Sci, 2017, 15 (376): 112-116.

[10] Chinese Medical Association Neurology Society and Chinese Medical Association Neurology Branch of Cerebrovascular Disease. Guidelines for the diagnosis and treatment of Acute Ischemic Stroke in China 2014 [J]. 2015, (4): 246-257.

[11] Y Zhao, W Yang, Z Tan, et al. Clopidogrel loading dose versus maintenance dose to treat patients with acute is chaemic stroke in China (CLASS-China): results from a prospective double-blind randomised clinical trial. Stroke Vasc Neurol, 2017, 2 (3): 118-123.

[12] A Zhang, X Hu, L Yang, et al. Combined analysis of CYP2C19 genotype incidence in Asian healthy population [J], Chinese Journal of evidence based medicine, 2014, 14 (4): 427-34.

[13] R Han, Y Li. Study on the CYP2C19 gene polymorphism and clinical efficacy of clopidogrel in patients with stroke. Neural Injury and Functional Reconstruction, 2016 (6): 480-482.

[14] Gilard M, Arnaud B, Cornily JC, et al. Influence of omeprazole on the antiplatelet action of clopidogrel associated with aspirin: the randomized, double-blind OCLA (Omeprazole CLopidogrel Aspirin) study [J]. J Am Coll Cardiol, 2008, 51 (3): 256-260.

[15] Juurlink DN, Gomes T, Ko DT, et al. A population-based study of the drug interaction between proton pump inhibitors and clopidogrel [J]. CMAJ, 2009, 180 (7): 713-718.

[16] Y Wang, Y Wang, X Zhao, et al. Clopidogrel with aspirin in acute minor stroke or transient ischemic attack. N Engl J Med, 2013, 369 (1): 11-9. 